\title{
An unusual case of lymphoplasmacytic lymphoma/Waldenström macroglobulinemia presenting with intractable seizures and interference with automated testing
}

\author{
Kritika Krishnamurthy $^{1}$ (D) Vathany Sriganeshan ${ }^{2} \cdot$ Ana Maria Medina ${ }^{1,2}$ \\ Received: 30 June 2020 / Accepted: 6 December 2020 / Published online: 7 January 2021 \\ (C) The Author(s), under exclusive licence to Springer-Verlag GmbH, DE part of Springer Nature 2021
}

\begin{abstract}
Lymphoplasmacytic lymphoma (LPL) is a B cell neoplasm composed of small lymphocytes, plasmacytoid lymphocytes, and plasma cells, which typically involves the bone marrow. Most cases of LPL present with Waldenstrom macroglobulinemia (WM) defined as the presence of monoclonal immunoglobulins of IgM type and LPL in the bone marrow. Herein, we present a case of LPL/WM presenting with intractable refractory seizures. A 71-year-old man with a 5-month history of seizures was brought to the emergency room after being found unresponsive. Automated hematology analysis revealed imprecise counts, wide variation, and multiple error flags. Peripheral smear review showed extracellular pale blue clumps of amorphous material. A marked cryoprecipitate of $7 \%$ was detected on a cryoglobulin screen. Serum protein electrophoresis (SPEP) detected a M-protein $(1.58 \mathrm{~g} / \mathrm{dL})$ in the gamma region. Serum immunofixation revealed an IgM lambda monoclonal protein. A bone marrow biopsy showed hypercellularity with increased lymphocytes forming interstitial aggregates, admixed with plasmacytoid lymphocytes and plasma cells. Immunohistochemically, the lymphoma cells were positive for CD20 and negative for CD3, CD5, CD10, CD23, Cyclin D1 (Bcl-1), and Bcl-6. CD138 highlighted increased plasma cells. Flow cytometric analysis revealed 2.7\% B cells with a predominance of lambda light chains, suggestive of a monoclonal B cell population. MYD88 mutational analysis revealed c.794T>C (p.L265P) mutation. A diagnosis of lymphoplasmacytic lymphoma/Waldenstrom macroglobulinemia was established. The patient underwent plasmapheresis leading to significant improvement in his symptoms. This case highlights a rare, unusual presentation of LPL/WM where the interference in automated testing led to identification of cryoglobulinemia and subsequent diagnosis of LPL/WM.
\end{abstract}

Keywords Lymphoplasmacytic lymphoma/Waldenstrom macroglobulinemia $\cdot$ Cryoglobulinemia $\cdot$ Automated lab testing $\cdot$ CNS presentations of LPL/WM $\cdot$ MYD88L265P

\section{Introduction}

Lymphoplasmacytic lymphoma (LPL) is a B cell neoplasm composed of small lymphocytes, plasmacytoid lymphocytes, and plasma cells, which typically involves the bone marrow and sometimes the lymph nodes, spleen, and other extranodal sites $[1,2]$. Most cases of LPL present with Waldenstrom

Kritika Krishnamurthy

kritikakrishnamurthy@yahoo.com

1 A.M. Rywlin, MD Department of Pathology, Mount Sinai Medical Center, 4300, Alton road, Suite 2400, Miami Beach, FL 33140, USA

2 Herbert Wertheim College of Medicine, Florida International University, Miami, FL, USA macroglobulinemia (WM), defined as the presence of monoclonal immunoglobulins of IgM type and LPL in the bone marrow. LPL/WM is relatively rare, with an incidence of approximately $5 / 1,000,000 /$ year [3]. The median age at diagnosis is 70 years, with a slight male preponderance [3]. It is occasionally difficult to distinguish LPL from other small B cell lymphoid neoplasms, such as marginal zone lymphoma, or multiple myeloma. The presence of MYD88 L265P mutation helps in establishing the diagnosis in these instances $[4,5]$.

LPL/WM usually presents as an indolent lymphoma without any disease-specific clinical features, although bone marrow failure and general B-symptoms often occur [6]. Patients usually present with vague clinical symptoms attributable to paraproteins produced and secreted by the malignant clone. Paraproteins, most often monoclonal IgM but rarely IgG or IgA, may cause hyperviscosity, autoantibody, or cryoglobulin 
activity, resulting in varied clinical manifestations, including neuropathy, diarrhea, and coagulopathy [7].

Herein, we present a case of LPL/WM presenting with intractable refractory seizures. The diagnosis of LPL/WM was suspected due to imprecise test results on automated testing of the patient's blood samples which prompted a peripheral smear review and identification of cryoglobulins on the peripheral smear, leading to a workup and subsequent diagnosis of LPL/WM.

\section{Clinical history}

The patient is a 71-year-old hypertensive man with a 5-month history of seizures who was brought to the emergency room after being found unresponsive. He was found to be hypotensive and in acute respiratory failure. His CT head failed to reveal any acute intracranial abnormalities and a carotid Doppler did not reveal any significant stenosis or plaque formation. His EKG and echocardiogram were within normal limits. Only significant lab abnormalities were a mild neutrophilia and a mild reversal in the serum albumin: globulin ratio $(3.7 / 4.4 \mathrm{~g} / \mathrm{dL})$. He was admitted to the intensive care unit for further management. A detailed history was elicited from his wife. She traced the patient's history of seizures to a similar episode of syncope 6 months prior when he fell and hit his head. At that time, he was taken to the emergency room and was found to be hypotensive. His CT head at that time had also failed to reveal any acute intracranial abnormalities. His EKG and echocardiogram were also within normal limits. In the absence of any significant intracranial abnormality, his fainting episode was attributed to his anti-hypertensive medication. Soon after, he started having episodes of staring into space accompanied by generalized tonic-clonic activity. His post-seizure period was marked with disorientation and confusion for approximately 20-30 min. The patient underwent brain imaging, EEG, and other workup which failed to reveal any significant intracranial abnormality that could explain the seizures. He was treated with levetiracetam and oxcarbazepine but was eventually switched over to phenytoin because of increasing frequency of seizures.

\section{Materials and methods}

Blood counts were performed on Sysmex XN 3100 automated analyzers. The red blood cell (RBC) and platelet count were performed by hemodynamically focused impedance measurements, while the white blood cell (WBC) differential and reticulocyte count were performed by fluorescence flow cytometry.

The serum protein electrophoresis (SPEP) and immunofixation was performed on Sebia capillary sys 2 and hydrasys 2 automated systems. The flow cytometry was performed on Beckman Coulter cytomics FC500 5-color bench top automated flow cytometer. Immunohistochemical studies were performed in all cases using the primary antibody anti-CD 20 (L26), anti-kappa, anti-lambda, anti-CD3 (2GV6), anti-CD 5 (SP19), anti-CD10 (SP67) and anti-CD23 (SP23) (Ventana Medical Systems, Inc., Tucson, AZ, USA), anti-CD138 (Clone MI15), anti-human Cyclin D1 (SP4) and anti-Bcl6 (PG-B6p) (Agilent technologies, Inc., Santa Clara, CA, USA), Ventana Benchmark automated slide stainer, and UltraView Universal Alkaline Phosphatase Red Detection Kit (Ventana Medical Systems, Inc., Tucson, AZ, USA).

MYD88 mutation analysis was performed by high sensitivity bidirectional Sanger sequencing of exon 5 of MYD 88 gene.

\section{Result}

Automated blood counts revealed hemoglobin of $6.2 \mathrm{~g} / \mathrm{dL}$ with possible interference error flag. Repeat automated hematology analysis on the same specimen revealed lack of precision with hemoglobin, total white blood cell counts, and platelet counts showing wide variation and multiple error flags including distribution width flag for RBC measurement, abnormal scattergram for WBC measurement, and hemoglobin turbidity error flag. Grossly, the specimen showed a distinct layering on sedimentation, with a lowest layer composed of cellular components, a second layer of thick, viscous, jelly like fluid, and a topmost layer of plasma (Fig. 1a). Peripheral smear review showed adequate numbers of normocytic, normochromic RBCs. Extracellular pale blue clumps of amorphous material were noted (Fig. 1b).

Cryoglobulinemia was suspected. A marked cryoprecipitate of 7\% was detected on a cryoglobulin screen. Immunofixation studies of this cryoprecipitate revealed an IgM lambda monoclonal protein migrating in the gamma region consistent with type I cryoglobulin.

SPEP detected a low level M-protein $(0.26 \mathrm{~g} / \mathrm{dL})$ in the gamma region (Fig. 1c). This peak increased in size $(1.58 \mathrm{~g} /$ dL) when the SPEP was performed at $37^{\circ} \mathrm{C}$ (Fig. 1d). Serum immunofixation revealed an IgM lambda monoclonal protein (Fig. 1e).

A bone marrow biopsy showed hypercellularity with increased lymphocytes forming interstitial aggregates, admixed with plasmacytoid lymphocytes and plasma cells (Fig. 1f) with Dutcher bodies (Fig. 1g). Immunohistochemically, the lymphoma cells were positive for CD20 (Fig. 1h) and negative for CD3, CD5, CD10, CD23, Cyclin D1 (Bcl-1,) and Bcl-6. CD138 (Fig. 1i) highlighted increased plasma cells.

Flow cytometric analysis revealed $2.7 \% \mathrm{~B}$ cells with a predominance of lambda light chains, suggestive of a monoclonal B cell population. MYD88 mutational analysis revealed c.794T $>$ C (p.L265P) mutation. A diagnosis of lymphoplasmacytic lymphoma/Waldenstrom macroglobulinemia was established. 


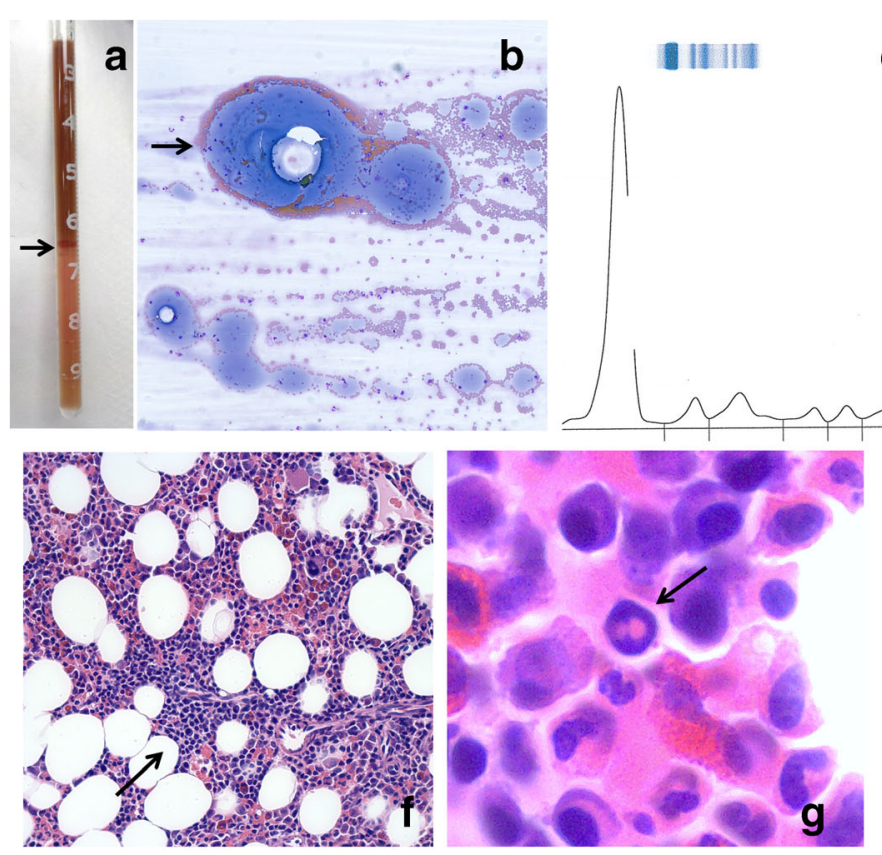

Fig. 1 a Distinct layering on gross examination marked by black arrow, with a lowest layer composed of cellular components, a second layer of thick, viscous, jelly like fluid, and a topmost layer of plasma. b Extracellular pale blue clumps of amorphous material on peripheral smear. c Serum protein electrophoresis (SPEP) detected a low level Mprotein $(0.26 \mathrm{~g} / \mathrm{dL})$ in the gamma region. $\mathbf{d}$ The peak was higher $(1.58 \mathrm{~g} /$ dL) when the SPEP was performed at $37^{\circ} \mathrm{C}$. e Serum immunofixation revealed an IgM lambda monoclonal protein. f Hematoxylin and eosin

The patient underwent plasmapheresis leading to significant improvement in his symptoms including cessation of seizures. The patient was started on chemotherapy with low dose bendamustine. He is being followed up as an outpatient and reports feeling much better with resolution of symptoms.

\section{Discussion}

LPL is an indolent lymphoproliferative neoplasm characterized by mixed population of small B lymphocytes, plasmacytoid lymphocytes, and plasma cells involving the bone marrow, lymph nodes, and spleen. WM is a subset of LPL with bone marrow involvement and detectable IgM monoclonal gammopathy, originally described in 1944 by Jan G. Waldenstrom [8-10]. LPL/WM is a relatively rare disease, with an annual incidence of less than 5 cases per million people, most commonly seen in older, white men $[11,12]$.

Peripheral blood examination may reveal lymphocytosis with primarily small circulating neoplastic cells having condensed nuclear chromatin and inconspicuous nucleoli. A subset of plasmacytoid lymphocytes, characterized by eccentric nuclei and abundant basophilic cytoplasm, is typically identified. $\mathrm{RBC}$ rouleaux formation may be seen. Bone marrow infiltration may display various patterns including diffuse, interstitial
C


stained section of bone marrow showing hypercellularity with increased lymphocytes forming interstitial aggregates, admixed with plasmacytoid lymphocytes and plasma cells $(\times 40$ objective, $\times 400$ magnification). $\mathbf{g}$ Dutcher bodies seen on hematoxylin and eosin stained bone marrow sections $(\times 100$ objective with oil, $\times 1000$ magnification $)$. h The lymphoma cells were positive for CD20 ( $\times 20$ objective, $\times 200$ magnification $)$. i Anti-CD138 highlighted increased plasma cells $(\times 20$ objective, $\times 200$ magnification)

as seen in our case, or focal non-paratrabecular. A solely paratrabecular pattern of infiltration is unusual [13]. Increased mast cells are often present and may play a supportive role in growth of neoplastic cells [14]. Accumulation of cytoplasmic or pseudonuclear immunoglobulin in the plasma cells in the form of Russell bodies and Dutcher bodies are typically present [13].

Patients are mostly asymptomatic but may present with pancytopenia, organomegaly, and hyperviscosity which are manifestations of infiltration of the bone marrow and extramedullary sites, such as the lymph nodes, spleen, and liver by malignant B cells and elevated IgM levels [15]. Characterization of the paraprotein is essential in the laboratory workup of LPL/WM. Serum protein electrophoresis, along with a densitometry tracing of the pattern, demonstrate a monoclonal peak (M-spike) in the gamma region. Immunofixation helps further characterize the type of heavy and light chain [13].

These paraproteins may exhibit autoantibody or cryoglobulinic activity and can cause hyperviscosity. It is the paraprotein activity that leads to the varied clinical manifestations of LPL/WM [7]. Cryoglobulins are proteins that precipitate at temperatures $<37^{\circ} \mathrm{C}$. Environmental cooling of the blood from venipuncture to analysis may have been sufficient for the cryoglobulins to precipitate, thus interfering with WBC and platelet counts in the analyzers. The degree of 
interference depends on the size, structure, and shape of the cryoglobulin precipitate aspirated into the measurement column. This is a possible explanation for the interference with automated testing seen in our case. The underestimation of hemoglobin seen in our case may be explained by a decreased $\mathrm{RBC}$ count because of a flow disorder. The presence of numerous thin precipitates of cryoglobulins in blood probably clogged the uptake system of the analyzer resulting in erroneous results. Examination of the peripheral blood revealed adequate normocytic, normochromic RBCs. Reliable counts can be obtained by warming the blood specimen at $37{ }^{\circ} \mathrm{C}$ for at least $30 \mathrm{~min}$. The temperature sensitivity of cryoglobulins also explains the significantly higher M-protein spike obtained on electrophoresis at $37^{\circ} \mathrm{C}$ in our case.

Cryoglobulinemia may present with immune complexmediated vasculitis usually affecting small to medium-sized vessels [16]. Manifestations include skin lesions including purpura and Raynaud phenomenon, arthralgia, peripheral neuropathy, and glomerulonephritis [17].

Symptoms of hyperviscosity generally start manifesting at serum viscosity of 4 to $5 \mathrm{cP}$ (corresponding to a serum IgM level of at least $3.0 \mathrm{~g} / \mathrm{dL}$ ) $[18,19]$. Hyperviscosity syndrome occurs in up to $30 \%$ of patients and may present with mucosal bleeding, visual disturbance, headache, or severe neurologic manifestations. García-Sanz et al. and Levine et al. reported neurologic manifestations to be a common presentation of WM, occurring in $22 \%$ and $48 \%$ of the studied patient populations, respectively. Peripheral neuropathy involving mostly the sensory nerves has been reported to be the predominant presentation [20-22]. In our case, the patient presented with central nervous system (CNS) manifestations in the form of seizures attributable to paraproteinemia at much lower serum IgM concentrations of $1.58 \mathrm{~g} / \mathrm{dL}$. It is possible that the serum IgM concentration may have been significantly higher than that recorded but was masked by incomplete disaggregation of cryoglobulin even at $37^{\circ} \mathrm{C}$.

CNS manifestations of cryoglobulinemia have been described only infrequently. Cacoub et al. reported the frequency of CNS complications to be 15 times less than peripheral neuropathy [23]. Feldman et al. reported CNS complications in less than $10 \%$ of cryoglobulinemic patients with neurological complications and less than one-third of these cases presented with seizures [24]. Very few studies address the exact underlying mechanism of seizures in cryoglobulinemia, but many authors including Cacoub et al. suggest CNS small vessel vasculitis as one of the underlying mechanism of the seizures. The cryoglobulinic provenance explains why the seizures did not respond to conventional anti-epileptic treatment but respond dramatically to plasmapheresis leading to removal of immunoglobulin complexes, as seen in our case. [25, 26]

Treatment of LPL/WM is generally expectant in asymptomatic patients. Symptomatic patients with modest hematologic abnormalities, IgM-related neuropathy, hemolytic anemia, or glomerulonephritis can be treated with singleagent rituximab therapy without maintenance. Patients with bulky disease, profound cytopenias, constitutional symptoms, and hyperviscosity syndrome are typically treated with emergent plasmapheresis, a regimen of rituximab, cyclophosphamide, and dexamethasone [13].Novel therapeutic agents targeting dysregulated signaling pathways, such as proteasome and PI3K/Akt $/ \mathrm{mTOR}$, are under evaluation for treatment of LPL/WM [27].

In summary, LPL is a B cell neoplasm composed of small lymphocytes, plasmacytoid lymphocytes, and plasma cells. Most cases of LPL are WM, with bone marrow involvement and detectable monoclonal IgM serum paraprotein. LPL/WM presenting with isolated CNS manifestations is extremely rare and hard to recognize. This case highlights a rare, unusual presentation of LPL/WM where the interference in automated testing led to identification of cryoglobulinemia and subsequent diagnosis of LPL/WM.

Data availability All data included

\section{Compliance with ethical standards}

Conflict of interest The authors declare that they have no conflict of interest.

Code availability NA

\section{References}

1. Swerdlow SH, Berger F, Pileri SA, Harris NL, Jaffe ES, Stein H (2017) Lymphoplasmacytic lymphoma. In: Swerdlow SH, Campo E, Harris NL et al (eds) WHO Classification of tumours of haematopoietic and lymphoid tissues, Revised 4th edn. International Agency for Research on Cancer, Lyon, pp 232-234

2. Dimopoulos MA, Kyle RA, Anagnostopoulos A, Treon SP (2005) Diagnosis and management of Waldenstrom macroglobulinemia. $\mathrm{J}$ Clin Oncol 23:1564-1577

3. Mazzucchelli M, Frustaci AM, Deodato M, Cairoli R, Tedeschi A (2018) Waldenstrom macroglobulinemia: an update. Mediterr J Hematol Infect Dis 10:e2018004

4. Treon SP, Xu L, Yang G, Zhou Y, Liu X, Cao Y, Sheehy P, Manning RJ, Patterson CJ, Tripsas C, Arcaini L, Pinkus GS, Rodig SJ, Sohani AR, Harris NL, Laramie JM, Skifter DA, Lincoln SE, Hunter ZR (2012) MYD88 L265P somatic mutation in Waldenstrom macroglobulinemia. N Engl J Med 367:826-833

5. Varettoni M, Arcaini L, Zibellini S, Boveri E, Rattotti S, Riboni R, Corso A, Orlandi E, Bonfichi M, Gotti M, Pascutto C, Mangiacavalli S, Croci G, Fiaccadori V, Morello L, Guerrera ML, Paulli M, Cazzola M (2013) Prevalence and clinical significance of the MYD88 (L265P) somatic mutation in Waldenstrom macroglobulinemia and related lymphoid neoplasms. Blood 121: 2522-2528

6. Gertz MA (2019) Waldenstrom macroglobulinemia: 2019 update on diagnosis, risk stratification, and management. Am J Hematol 94:266-276

7. Fukushima M, Okoshi Y, Fukazawa K, Koshino M, Ishiguro S, Mitsuhashi S, Saitoh H, Iijima T, Kojima H, Hori M (2017) 
Lymphoplasmacytic lymphoma presenting with diarrhea and joint pain which was successfully diagnosed by an MYD88 mutation analysis. Intern Med 56(7):847-851

8. Owen RG, Treon SP, Al-Katib A et al (2003) Clinicopathological definition of Waldenstrom macroglobulinemia: consensus panel recommendations from the Second International Workshop on Waldenstr "om's macroglobulinemia. Semin Oncol 30(2):110-115

9. Swerdlow SH, Campo E, Harris NL, et al, eds (2008) WHO classification of tumours of the haematopoietic and lymphoid tissues. 4th ed. Lyon, France:IARC Press. World Health Organization Classification of Tumours; vol 2

10. Waldenstorm J (1944) Incipient myelomatosis or 'essential' hyperglobulinemia with fibrinogenopenia: a new syndrome? Acta Med Scand 17(3):216-222

11. Groves FD, Travis LB, Devesa SS, Ries LA, Fraumeni JF Jr (1998) Waldenstrom macroglobulinemia: incidence patterns in the United States, 1988-1994. Cancer. 82(6):1078-1081

12. Herrinton LJ, Weiss NS (1993) Incidence of Waldenstrom macroglobulinemia. Blood. 82(10):3148-3150

13. Naderi N, Yang DT (2013) MDLymphoplasmacytic lymphoma and Waldenstrom Macroglobulinemia. Arch Pathol Lab Med 137: $580-585$

14. Tournilhac O, Santos DD, Xu L, Kutok J, Tai YT, le Gouill S, Catley L, Hunter Z, Branagan AR, Boyce JA, Munshi N, Anderson KC, Treon SP (2006) Mast cells in Waldenstrom macroglobulinemia support lymphoplasmacytic cell growth through CD154/CD40 signaling. Ann Oncol 17(8):1275-1282

15. Bjorkholm M, Johannsson E, Papamichael D et al (2003) Patterns of clinical presentation, treatment, and outcome in patients with Waldenstrom macroglobulinemia: a two-institution study. Semin Oncol 30(2):226-230

16. Aoki H, Takishita M, Kosaka M, Saito S (1995) Frequent somatic mutations in D and/or JH segments of ig gene in Waldenstrom macroglobulinemia and chronic lymphocytic leukemia (CLL) with Richter's syndrome but not in common CLL. Blood. 85(7):1913-1919

17. Khan TMA, Ansari Y, Siddiqui AH, Ansari SA, Siddiqui F (2019) Development of central nervous system vasculitis in a patient with
Waldenstrom macroglobulinemia: a rare presentation with poor prognosis. Cureus. 11(10):e6039

18. Crawford J, Cox EB, Cohen HJ (1985) Evaluation of hyperviscosity in monoclonal gammopathies. Am J Med 79(1):13-22

19. MacKenzie MR, Lee TK (1977) Blood viscosity in Waldenstrom macroglobulinemia. Blood. 49(4):507-510

20. García-Sanz R, Montoto S, Torrequebrada A, de Coca AG, Petit J, Sureda A, Rodríguez-García JA, Massó P, Pérez-Aliaga A, Monteagudo MD, Navarro I, Moreno G, Toledo C, Alonso A, Besses C, Besalduch J, Jarque I, Salama P, Rivas JA, Navarro B, Bladé J, Miguel JF, Spanish Group for the Study of Waldenström Macroglobulinaemia and PETHEMA (Programme for the Study and Treatment of Haematological Malignancies) (2001) Waldenström macroglobulinaemia: presenting features and outcome in a series with 217 cases. Br J Haematol 115:575-582

21. Dimopoulos MA, Anagnostopoulos A (2005) Waldenström macroglobulinemia. Best Pract Res Clin Haematol 18:747-765. https:// doi.org/10.1016/j.beha.2005.01.028

22. Baehring JM, Hochberg EP, Raje N, Ulrickson M, Hochberg FH (2008) Neurological manifestations of Waldenstrom macroglobulinemia. Nat Clin Pract Neurol 4:547-556

23. Cacoub P, Comarmond C, Domont F, Savey L, Saadoun D (2015) Cryoglobulinemia vasculitis. Am J Med 128:950-955

24. Feldman L, Dhamne M, Li Y (2019) Neurologic manifestations associated with cryoglobulinemia: a single center experience. J Neurol Sci 398:121-127

25. Reik L, Korn JH (1981) Cryoglobulinemia with encephalopathy: successful treatment by plasma exchange. Ann Neurol 10:488-490

26. Pagano G, Komici K, Femminella GD, de Lucia C, Ianniciello M, Leosco D, Ferrara N, Rengo G (2014) Sudden onset of coma in a 70year-old woman with cryoglobulinemia. Am J Case Rep 15:56-59

27. Issa GC, Leblebjian H, Roccaro AM, Ghobrial IM (2011) New insights into the pathogenesis and treatment of Waldenstrom macroglobulinemia. Curr Opin Hematol 18(4):260-265

Publisher's note Springer Nature remains neutral with regard to jurisdictional claims in published maps and institutional affiliations. 